\title{
Sampled-Data Consensus of Second-Order Multi-Agent Systems with Delayed-State-Derivative Feedback
}

\author{
Na Wang, Zhihai Wu and Li Peng \\ The Key Laboratory for Advanced Process Control of Light Industry of the Ministry of Education, School of Internet \\ of Things Engineering Jiangnan University, Wuxi, 214122, P. R. China \\ E-mail:wuzhihai@jiangnan.edu.cnｗfwangna@126.com
}

\begin{abstract}
This paper is concerned with sampled-data consensus of second-order delayed multi-agent systems with delayed-statederivative feedback. First, the delay decomposition technique is used for obtaining the consensus protocol based on sampled-data. Then, the stability theory of linear systems and algebra graph theory are employed to derive the necessary and sufficient conditions about the sampling period guaranteeing the achievement of stationary consensus. Last, simulations are provided to demonstrate the effectiveness of the theoretical results.
\end{abstract}

Index Terms - Second-Order Multi-Agent Systems, Consensus, Delayed-State-Derivative Feedback, Sampled-Data.

\section{Introduction}

Recently, consensus of multi-agent systems, due to its broad applications in many areas including cooperative control of unmanned air vehicles, automated highway systems, swarming, flocking, rendezvous, formation control, distributed sensor networks and congestion control in communication networks, has attracted a great deal of attention in various fields such as physics, biology, robotics and control engineering. In the past decade, considerable efforts have been devoted to consensus problems. Vicsek et al. proposed a simple model for phase transition of a group of self-driven particles and numerically demonstrated complex dynamics of the model [1]. Jadbabaie et al. provided a theoretical explanation for the consensus behavior of the Vicsek model using graph theory [2]. Ren and Beard extended the results of [2] to the directed topology case and gave some more relaxed topology conditions [3]. Olfati-Saber et al. provided a theoretical framework for the analysis of consensus algorithms for multi-agent systems, an overview of basic concepts of information consensus in networks and the methods of convergence analysis for the algorithms [4]. More results can be seen in the survey [5], [6] and the references therein.

For most of the existing consensus protocols, only current states or delayed ones are used [7]-[10]. However, in certain cases, the derivatives of delayed states are necessary to guarantee the achievement of consensus. In [11], Cao et al. investigated consensus tracking problems of the first-order and second-order continuous-time multi-agent systems with the time-varying reference state, proving that when the virtual leader is available to only a portion of agents, the proposed consensus tracking protocol without delay-state-derivative feedback cannot guarantee the achievement of consensus tracking, whereas the proposed consensus tracking protocol with delay-state-derivative feedback can do. Moreover, for continuous-time multi-agent systems with communication delay, introducing delay-state-derivative feedback with the proper feedback gain can improve the consensus performance including the robustness against communication delay and the convergence speed of achieving the consensus [12]-[15]. However, just as all the authors of references [11]-[15] pointed out, in real applications, it is not easy for an agent to obtain the delay-state-derivative information of its neighborhood agents, i.e., it is hard to implement the continuous-time consensus protocols with delay-state- derivative feedback. In order to implement the consensus protocols with delay-state-derivative feedback, all the authors of references [11]-[15] proposed the strategy of approximately calculating the derivatives of delayed states by using the numerical differentiations in the case of sampled-data control. However, this approximate calculation might destroy the achievement of consensus, and the strategy of choosing the sampling period for guaranteeing the achievement of consensus has been not provided in [11][15].

Based on the above consideration, the goal of this paper is to find conditions about the sampling period for the achievement of consensus of multi-agent systems with delaystate-derivative feedback based on sampled-data.

\section{Preliminaries and Problem Statement}

A. Algebra Graph Theory and Some Notations

Let $G=(V, E, A)$ be a weighted undirected graph with the set of nodes $V=\left\{v_{1}, v_{2}, \cdots, v_{N}\right\}$, the set of edges $E \subseteq V \times V$ and the weighted adjacency matrix $A=\left[a_{i j}\right]$ with nonnegative adjacency elements $a_{i j}$. The node indexes of $G$ belong to a finite index set $I=\{1,2, \cdots, N\}$. An edge of $G$ is denoted by $e_{i j}=\left(v_{i}, v_{j}\right)$. The adjacency elements associated with the edges are positive, i.e., $e_{i j} \in E \Leftrightarrow a_{i j}>0$.

\footnotetext{
* This work is supported by National Natural Science Foundation of China under Grant Nos. 61203147, 60973095, 60804013 and 61104092, Fundamental Research Funds for the Central Universities of China under Grant Nos. JUSRP111A44 and JUSRP21011, and Humanities and Social Sciences Youth Funds of the Ministry of Education under Grant No. 12YJCZH218.
} 
Moreover, we assume $a_{i i}=0$ for all $i \in I$. For the undirected graph $G$, the adjacency matrix $A$ is symmetric, i.e., $a_{i j}=a_{j i}$. The set of neighbors of node $v_{i}$ is denoted by $N_{i}=\left\{v_{j} \in V: e_{i j} \in E\right\}$. The degree of node $v_{i}$ is defined as $d_{i}=\sum_{j \in N_{i}} a_{i j}$. The Laplacian matrix of $G$ is defined as $L=D-A$, where $D=\operatorname{Diag}\left(d_{1}, d_{2}, \cdots, d_{N}\right)$ is the degree matrix of $G$ with diagonal elements $d_{i}$ and zero off-diagonal elements. An important fact of $L$ is that all the row sums are zero and thus $L$ has a right eigenvector $1_{N}$ associated with the zero eigenvalue, where $1_{N}$ denotes the $N$-dimensional column vector with all ones. A path between two distinct nodes $v_{i}$ and $v_{j}$ is meant a sequence of distinct edges of the form $\left(v_{i}, v_{k_{1}}\right),\left(v_{k_{1}}, v_{k_{2}}\right), \cdots,\left(v_{k_{l}}, v_{j}\right)$. A graph is called connected if there is a path between any two distinct nodes of the graph. An important property of the Laplacian matrix, which is instrumental in the convergence analysis of consensus protocols, is that the graph $G$ is connected if and only if $\operatorname{rank}(L)=N-1$ [16]. Thus, for a connected graph $G, L$ has one and only one zero eigenvalue and all the other eigenvalues of $L$ are positive. Without loss of generality, for a connected graph $G$, all the eigenvalues of $L$ can be ordered sequentially in an ascending order as $0=\lambda_{1}(L)<\lambda_{2}(L) \leq \cdots \leq \lambda_{N}(L)$.

The following notations will be used throughout this paper. $0_{N}$ denotes the $N$-dimensional column vectors with all zeros. $I_{N}$ denotes the $N$-dimensional identity matrix. Let $R$ denote the set of real numbers. For a given matrix $M$, $\operatorname{det}(M)$ denotes its determinant.

\section{B. Problem Statement}

In a multi-agent system with $N$ agents, an agent and an available information flow between two agents are considered as a node and an edge in an undirected graph, respectively. Consider the system of double-integrator dynamic agents described by

$$
\left\{\begin{array}{l}
\xi_{i}(t)=\zeta_{i}(t) \\
\xi_{i}(t)=u_{i}(t)
\end{array}, i \in I .\right.
$$

where $\xi_{i}(t) \in R$ and $\zeta_{i}(t) \in R$ represent, respectively, the position and the velocity of the $i$ th agent, and $u_{i}(t) \in R$ is the associated control input called the consensus protocol.

In order to simultaneously improve the robustness against communication delay and the convergence speed of achieving the consensus, in [13] $\mathrm{Wu}$ and Fang proposed the following consensus protocol with delay-state-derivative feedback:

$$
\begin{aligned}
u_{i}(t)= & -\gamma \zeta_{i}(t)+\sum_{j \in N_{i}} a_{i j}\left\{\gamma\left[\xi_{j}(t-\tau)-\xi_{i}(t-\tau)\right]\right. \\
& +(1+\gamma \beta)\left[\zeta_{j}(t-\tau)-\zeta_{i}(t-\tau)\right] \\
& \left.+\beta\left[\dot{\zeta}_{j}(t-\tau)-\dot{\zeta}_{i}(t-\tau)\right]\right\} .
\end{aligned}
$$

where $\tau>0$ represents communication delay between agents, $\gamma>0$ is the control parameter, and $\beta$ is the intensity of delaystate-derivative feedback and satisfies $\beta \in\left(0, \min \left\{\tau, 1 / \lambda_{N}(L)\right\}\right)$.

According to the suggestion in [11]-[15], while implementing the consensus protocol (2), $\dot{\zeta}_{i}(t-\tau)$ and $\dot{\zeta}_{j}(t-\tau), j \in N_{i}$ are replaced with $\left(\zeta_{i}(t-\tau)-\zeta_{i}(t-\tau-h)\right) / h$ and $\left(\zeta_{j}(t-\tau)-\zeta_{j}(t-\tau-h)\right) / h, j \in N_{i}$, respectively, where $h>0$ is the sampling period. Then we can get the following consensus protocol with numerical differentiation:

$$
\begin{aligned}
& u_{i}(t)=-\gamma \zeta_{i}(t)+\sum_{j \in N_{i}} a_{i j}\left\{\gamma\left[\xi_{j}(t-\tau)-\xi_{i}(t-\tau)\right]\right. \\
& +(1+\gamma \beta)\left[\zeta_{j}(t-\tau)-\zeta_{i}(t-\tau)\right] \\
& +\beta\left[\left(\zeta_{j}(t-\tau)-\zeta_{j}(t-\tau-h)\right) / h\right. \\
& \left.\left.-\left(\zeta_{i}(t-\tau)-\zeta_{i}(t-\tau-h)\right) / h\right]\right\} .
\end{aligned}
$$

Decomposing communication delay $\tau$ with respect to the sampling period $h$ yields

$$
\tau=m h+\varepsilon
$$

where $m$ is a nonnegative integer and $\varepsilon \in[0, h)$.

Using the period sampling technology and zero-order hold circuit, the following consensus protocol based on sampleddata is induced from the consensus protocol (3):

$$
u_{i}(t)=\left\{\begin{array}{l}
u_{i}(k h-m h-h)=-\gamma \zeta_{i}(k h-h)+ \\
\sum_{j \in N_{i}} a_{i j}\left\{\gamma\left[\xi_{j}(k h-m h-h)-\xi_{i}(k h-m h-h)\right]\right. \\
+(1+\gamma \beta)\left[\zeta_{j}(k h-m h-h)-\zeta_{i}(k h-m h-h)\right] \\
+\beta\left[\left(\zeta_{j}(k h-m h-h)-\zeta_{j}(k h-m h-2 h)\right) / h\right. \\
\left.\left.-\left(\zeta_{i}(k h-m h-h)-\zeta_{i}(k h-m h-2 h)\right) / h\right]\right\}, \\
t \in[k h, k h+\varepsilon) ; \\
u_{i}(k h-m h)=-\gamma \zeta_{i}(k h)+\sum_{j \in N_{i}} a_{i j}\left\{\gamma \left[\xi_{j}(k h-m h)-\right.\right. \\
\left.\xi_{i}(k h-m h)\right]+(1+\gamma \beta)\left[\zeta_{j}(k h-m h)-\zeta_{i}(k h-m h)\right] \\
+\beta\left[\left(\zeta_{j}(k h-m h)-\zeta_{j}(k h-m h-h)\right) / h\right. \\
\left.\left.-\left(\zeta_{i}(k h-m h)-\zeta_{i}(k h-m h-h)\right) / h\right]\right\}, \\
t \in[k h+\varepsilon, k h+h) .
\end{array}\right.
$$

It is said that the multi-agent system (1) asymptotically achieves the stationary consensus, if $\lim _{t \rightarrow \infty} \xi_{i}(t)=c$, $\lim _{t \rightarrow \infty} \zeta_{i}(t)=0, \forall i \in I$, where $c$ is a constant.

In the following, we will find the necessary and sufficient conditions guaranteeing the system (1) applying the consensus protocol (5) to achieve the stationary consensus. 


\section{Convergence Analysis}

In this section, employing the stability theory of linear systems and algebra graph theory, we derive the necessary and sufficient conditions guaranteeing the system (1) applying the consensus protocol (5) to achieve the stationary consensus. The main results are as follows:

Theorem 3.1: Consider the second-order multi-agent system (1) with a fixed, undirected and connected network topology. The multi-agent system (1), applying the consensus protocol (5), achieves the stationary consensus if and only if all the roots of the equation

$$
\begin{aligned}
& z^{m+4}+(h r-\varepsilon r-2) z^{m+3}+(1-h r+2 \varepsilon r) z^{m+2} \\
& -\varepsilon r z^{m+1}+\left[(h-\varepsilon)^{2} r / 2+h+h r \beta-\varepsilon-\varepsilon r \beta\right. \\
& +\beta-\varepsilon \beta / h] \lambda_{i}(L) z^{3}+\left(h^{2} r / 2-\varepsilon^{2} r+h r \varepsilon\right. \\
& -h-h r \beta+2 \varepsilon r \beta+2 \varepsilon-2 \beta+3 \varepsilon \beta / h) \lambda_{i}(L) z^{2} \\
& +\left(\varepsilon^{2} r / 2-\varepsilon-\varepsilon r \beta-3 \varepsilon \beta / h+\beta\right) \lambda_{i}(L) z \\
& +(\varepsilon \beta / h) \lambda_{i}(L)=0, i \in I \backslash\{1\} .
\end{aligned}
$$

are within the unit circle.

Proof: When $t \in[k h, k h+\varepsilon)$, from (1) and (5) we obtain

$$
\begin{aligned}
& \left(\begin{array}{l}
\xi_{i}(t) \\
\zeta_{i}(t)
\end{array}\right)=\exp \left\{\left[\begin{array}{ll}
0 & 1 \\
0 & 0
\end{array}\right](t-k h)\right\}\left(\begin{array}{l}
\xi_{i}(k h) \\
\zeta_{i}(k h)
\end{array}\right) \\
& +\int_{k h}^{t} \exp \left\{\left[\begin{array}{ll}
0 & 1 \\
0 & 0
\end{array}\right]\left(t-t^{\prime}\right)\right\}\left(\begin{array}{l}
0 \\
1
\end{array}\right) u_{i}(k h-m h-h) \mathrm{d} t^{\prime} \\
& =\left[\begin{array}{cc}
1 & t-k h \\
0 & 1
\end{array}\right]\left(\begin{array}{l}
\xi_{i}(k h) \\
\zeta_{i}(k h)
\end{array}\right)+\left(\begin{array}{c}
(t-k h)^{2} / 2 \\
t-k h
\end{array}\right) u_{i}(k h-m h-h)
\end{aligned}
$$

which further leads to

$$
\begin{aligned}
& \left(\begin{array}{c}
\xi_{i}(k h+\varepsilon) \\
\zeta_{i}(k h+\varepsilon)
\end{array}\right)=\left[\begin{array}{ll}
1 & \varepsilon \\
0 & 1
\end{array}\right]\left(\begin{array}{l}
\xi_{i}(k h) \\
\zeta_{i}(k h)
\end{array}\right) \\
& +\left(\begin{array}{c}
\varepsilon^{2} / 2 \\
\varepsilon
\end{array}\right) u_{i}(k h-m h-h), k=0,1,2, \cdots ; i \in I .
\end{aligned}
$$

Similarly, when $t \in[k h+\varepsilon, k h+h)$, from (1) and (5) we get

$$
\begin{aligned}
& \left(\begin{array}{l}
\xi_{i}(t) \\
\zeta_{i}(t)
\end{array}\right)=\exp \left\{\left[\begin{array}{ll}
0 & 1 \\
0 & 0
\end{array}\right](t-k h-\varepsilon)\right\}\left(\begin{array}{l}
\xi_{i}(k h+\varepsilon) \\
\zeta_{i}(k h+\varepsilon)
\end{array}\right) \\
& +\int_{k h+\varepsilon}^{t} \exp \left\{\left[\begin{array}{ll}
0 & 1 \\
0 & 0
\end{array}\right]\left(t-t^{\prime}\right)\right\}\left(\begin{array}{l}
0 \\
1
\end{array}\right) u_{i}(k h-m h) \mathrm{d} t^{\prime}= \\
& {\left[\begin{array}{cc}
1 & t-k h-\varepsilon \\
0 & 1
\end{array}\right]\left(\begin{array}{l}
\xi_{i}(k h+\varepsilon) \\
\zeta_{i}(k h+\varepsilon)
\end{array}\right)+\left(\begin{array}{c}
(t-k h-\varepsilon)^{2} / 2 \\
t-k h-\varepsilon
\end{array}\right) u_{i}(k h-m h)}
\end{aligned}
$$

which further results in

$$
\begin{aligned}
& \left(\begin{array}{c}
\xi_{i}(k h+h) \\
\zeta_{i}(k h+h)
\end{array}\right)=\left[\begin{array}{cc}
1 & h-\varepsilon \\
0 & 1
\end{array}\right]\left(\begin{array}{l}
\xi_{i}(k h+\varepsilon) \\
\zeta_{i}(k h+\varepsilon)
\end{array}\right) \\
& +\left(\begin{array}{c}
(h-\varepsilon)^{2} / 2 \\
\mathrm{~h}-\varepsilon
\end{array}\right) u_{i}(k h-m h), k=0,1,2, \cdots ; i \in I .
\end{aligned}
$$

Combining (8) and (10) yields the dynamics equation at the sampling instants:

$$
\left\{\begin{array}{l}
\xi_{i}(k h+h)=\xi_{i}(k h)+h \zeta_{i}(k h) \\
+\left(h \varepsilon-\varepsilon^{2} / 2\right) u_{i}(k h-m h-h) \\
+\left((h-\varepsilon)^{2} / 2\right) u_{i}(k h-m h) ; \\
\zeta_{i}(k h+h)=\zeta_{i}(k h)+\varepsilon u_{i}(k h-m h-h) \\
+(h-\varepsilon) u_{i}(k h-m h), k=0,1,2, \cdots ; i \in I .
\end{array}\right.
$$

Noticing $L 1_{N}=0_{N}$, we can rewrite the dynamics equation (11) in a compact form

$$
\left\{\begin{array}{l}
\xi(k h+h)=\xi(k h)+h \zeta(k h)+\left(h \varepsilon-\varepsilon^{2} / 2\right) \\
\times\{-\gamma \zeta(k h-h)-L[\gamma \xi(k h-m h-h) \\
+(1+\gamma \beta) \zeta(k h-m h-h)+\beta(\zeta(k h-m h-h) \\
-\zeta(k h-m h-2 h)) / h]\}+\left((h-\varepsilon)^{2} / 2\right)\{-\gamma \zeta(k h) \\
-L[\gamma \xi(k h-m h)+(1+\gamma \beta) \zeta(k h-m h) \\
+\beta(\zeta(k h-m h)-\zeta(k h-m h-h)) / h]\} ; \\
\zeta(k h+h)=\zeta(k h)+\varepsilon\{-\gamma \zeta(k h-h) \\
-L[\gamma \xi(k h-m h-h)+(1+\gamma \beta) \zeta(k h-m h-h) \\
+\beta(\zeta(k h-m h-h)-\zeta(k h-m h-2 h)) / h]\} \\
+(h-\varepsilon)\{-\gamma \zeta(k h)-L[\gamma \xi(k h-m h)+(1+\gamma \beta) \\
\times \zeta(k h-m h)+\beta(\zeta(k h-m h)-\zeta(k h-m h-h)) / h]\}, \\
k=0,1,2, \cdots ; i \in I .
\end{array}\right.
$$

where $\xi(k h)=\left(\xi_{1}(k h), \xi_{2}(k h), \cdots, \xi_{N}(k h)\right)^{T}$ and $\zeta(k h)=\left(\zeta_{1}(k h), \zeta_{2}(k h), \cdots, \zeta_{N}(k h)\right)^{T}$.

Taking z-transform of the system (12) leads to

$$
\left\{\begin{array}{l}
z \xi(z)=\xi(z)+h \zeta(z)+\left(h \varepsilon-\varepsilon^{2} / 2\right)\left\{-\gamma z^{-1} \zeta(z)\right. \\
-L z^{-(m+1)}[\gamma \xi(z)+(1+\gamma \beta) \zeta(z)+\beta(\zeta(z)- \\
\left.\left.\left.z^{-1} \zeta(z)\right) / h\right]\right\}+\left((h-\varepsilon)^{2} / 2\right)\left\{-\gamma \zeta(z)-L z^{-m}[\gamma \xi(z)\right. \\
\left.\left.+(1+\gamma \beta) \zeta(z)+\beta\left(\zeta(z)-z^{-1} \zeta(z)\right) / h\right]\right\} \\
z \zeta(z)=\zeta(z)+\varepsilon\left\{-\gamma z^{-1} \zeta(z)-L z^{-(m+1)}[\gamma \xi(z)\right. \\
\left.\left.+(1+\gamma \beta) \zeta(z)+\beta\left(\zeta(z)-z^{-1} \zeta(z)\right) / h\right]\right\}+(h-\varepsilon) \\
\times\left\{-\gamma \zeta(z)-L z^{-m}[\gamma \xi(z)+(1+\gamma \beta) \zeta(z)+\beta(\zeta(z)\right. \\
\left.\left.\left.-z^{-1} \zeta(z)\right) / h\right]\right\}, k=0,1,2, \mathrm{~L} ; i \in I .
\end{array}\right.
$$


where $\xi(z)$ and $\zeta(z)$ are the z-transform of $\xi(k h)$ and $\zeta(k h)$, respectively. From (13) we get the characteristic equation about $\xi(k h)$ as follows:

$$
\begin{aligned}
& z^{(m+2) N} \operatorname{det}\left\{\left[z^{m+4}+(h r-\varepsilon r-2) z^{m+3}+(1-h r\right.\right. \\
& \left.+2 \varepsilon r) z^{m+2}-\varepsilon r z^{m+1}\right] I_{N}+\left[\left((h-\varepsilon)^{2} r / 2+h+h r \beta\right.\right. \\
& -\varepsilon-\varepsilon r \beta+\beta-\varepsilon \beta / h) z^{3}+\left(h^{2} r / 2-\varepsilon^{2} r+h r \varepsilon-h\right. \\
& -h r \beta+2 \varepsilon r \beta+2 \varepsilon-2 \beta+3 \varepsilon \beta / h) z^{2}+\left(\varepsilon^{2} r / 2-\varepsilon\right. \\
& -\varepsilon r \beta-3 \varepsilon \beta / h+\beta) z+(\varepsilon \beta / h)] L\}=0 .
\end{aligned}
$$

Due to the connectivity of the network topology, (14) turns into

$$
z^{(m+2) N} \prod_{i=1}^{N}\left\{\begin{array}{l}
z^{m+4}+(h r-\varepsilon r-2) z^{m+3}+(1-h r \\
+2 \varepsilon r) z^{m+2}-\varepsilon r z^{m+1}+\left[\left((h-\varepsilon)^{2} r / 2\right.\right. \\
+h+h r \beta-\varepsilon-\varepsilon r \beta+\beta-\varepsilon \beta / h) z^{3} \\
+\left(h^{2} r / 2-\varepsilon^{2} r+h r \varepsilon-h-h r \beta+2 \varepsilon r \beta\right. \\
+2 \varepsilon-2 \beta+3 \varepsilon \beta / h) z^{2}+\left(\varepsilon^{2} r / 2-\varepsilon-\varepsilon r \beta\right. \\
-3 \varepsilon \beta / h+\beta) z+(\varepsilon \beta / h)] \lambda_{i}(L)
\end{array}\right\}=0 .
$$

Further simplifying (15) results in

$$
\begin{aligned}
& z^{(m+2) N+(m+1)}\left[z^{3}+(h r-\varepsilon r-2) z^{2}\right. \\
& +(1-h r+2 \varepsilon r) z-\varepsilon r] \\
& \times \prod_{i=2}^{N}\left\{\begin{array}{l}
z^{m+4}+(h r-\varepsilon r-2) z^{m+3}+(1-h r+2 \varepsilon r) z^{m+2} \\
-\varepsilon r z^{m+1}+\left[\left((h-\varepsilon)^{2} r / 2+h+h r \beta-\varepsilon-\varepsilon r \beta\right.\right. \\
+\beta-\varepsilon \beta / h) z^{3}+\left(h^{2} r / 2-\varepsilon^{2} r+h r \varepsilon-h-h r \beta\right. \\
+2 \varepsilon r \beta+2 \varepsilon-2 \beta+3 \varepsilon \beta / h) z^{2}+\left(\varepsilon^{2} r / 2-\varepsilon\right. \\
-\varepsilon r \beta-3 \varepsilon \beta / h+\beta) z+(\varepsilon \beta / h)] \lambda_{i}(L)
\end{array}\right\}=0
\end{aligned}
$$

It is obvious that (16) has only one root equal to 1 and $(m+2) N+(m+1)$ roots equal to 0 . Therefore, the system (12) is asymptotically stable if and only if all the roots of the equation (6) are within the unit circle.

Now it is assumed that all the roots of the equation (6) are within the unit circle. Denote the static state that the state $\xi_{i}(t)$ of the system ( 12 ) converges to as $\xi_{i}^{*}$, i.e., $\lim _{k \rightarrow \infty} \xi_{i}(k h)=\xi_{i}^{*}, \forall i \in I$ and

$$
\lim _{k \rightarrow \infty} \zeta_{i}(k h)=0, \forall i \in I .
$$

Thus, it is obtained from (12) that $L\left(\xi_{1}^{*}, \xi_{2}^{*}, \cdots, \xi_{N}^{*}\right)^{T}=0$. Notice that $L 1_{N}=0_{N}$, thus $\left(\xi_{1}^{*}, \xi_{2}^{*}, \cdots, \xi_{N}^{*}\right)^{T}=c 1_{N}$, i.e.,

$$
\lim _{k \rightarrow \infty} \xi_{i}(k h)=c, \forall i \in I .
$$

It follows from (7) that when $t \in[k h, k h+\varepsilon)$,

$$
\begin{aligned}
& \left(\begin{array}{l}
\xi(t) \\
\zeta(t)
\end{array}\right)-\left[\begin{array}{cc}
1 & t-k h \\
0 & 1
\end{array}\right]\left(\begin{array}{l}
\xi(k h) \\
\zeta(k h)
\end{array}\right) \\
& =\left(\begin{array}{c}
(t-k h)^{2} / 2 \\
t-k h
\end{array}\right)\{-\gamma \zeta(k h-h) \\
& +L[\gamma \xi(k h-m h-h)+(1+\gamma \beta) \zeta(k h-m h-h) \\
& +\beta(\zeta(k h-m h-h)-\zeta(k h-m h-2 h)) / h]\} .
\end{aligned}
$$

From (19) we get that when $t \in[k h, k h+\varepsilon)$,

$$
\left\{\begin{array}{l}
\lim _{t \rightarrow \infty}(\xi(t)-\xi(k h))=0 \\
\lim _{t \rightarrow \infty}(\zeta(t)-\zeta(k h))=0
\end{array}\right. \text {. }
$$

Similarly, from (8) and (9) we get that when $t \in[k h+\varepsilon, k h+h)$,

$$
\begin{aligned}
& \left(\begin{array}{l}
\xi(t) \\
\zeta(t)
\end{array}\right)-\left[\begin{array}{cc}
1 & t-k h \\
0 & 1
\end{array}\right]\left(\begin{array}{l}
\xi(k h) \\
\zeta(k h)
\end{array}\right) \\
& =\left(\begin{array}{c}
\left(\varepsilon^{2} / 2\right)+\varepsilon(t-k h-\varepsilon) \\
\varepsilon
\end{array}\right)\{-\gamma \zeta(k h-h) \\
& +L[\gamma \xi(k h-m h-h)+(1+\gamma \beta) \zeta(k h-m h-h) \\
& +\beta(\zeta(k h-m h-h)-\zeta(k h-m h-2 h)) / h]\} \\
& +\left(\begin{array}{c}
(t-k h-\varepsilon)^{2} / 2 \\
t-k h-\varepsilon
\end{array}\right)\{-\gamma \zeta(k h)+L[\gamma \xi(k h-m h) \\
& +(1+\gamma \beta) \zeta(k h-m h) \\
& +\beta(\zeta(k h-m h)-\zeta(k h-m h-h)) / h]\} .
\end{aligned}
$$

From (21) we get that when $t \in[k h+\varepsilon, k h+h)$,

$$
\left\{\begin{array}{l}
\lim _{t \rightarrow \infty}(\xi(t)-\xi(k h))=0 \\
\lim _{t \rightarrow \infty}(\zeta(t)-\zeta(k h))=0
\end{array} .\right.
$$

Combining (20) and (22) leads to

$$
\left\{\begin{array}{l}
\lim _{t \rightarrow \infty}(\xi(t)-\xi(k h))=0 \\
\lim _{t \rightarrow \infty}(\zeta(t)-\zeta(k h))=0
\end{array}, t \in[k h, k h+h) .\right.
$$

Combining (17), (18), and (23) results in

$$
\left\{\begin{array}{l}
\lim _{t \rightarrow \infty} \xi_{i}(t)=c \\
\lim _{t \rightarrow \infty} \zeta_{i}(t)=0
\end{array}, \forall i \in I\right.
$$

Based on the above analysis, the multi-agent system (1), applying the consensus protocol (5), achieves the stationary consensus if and only if all the roots of the equation (6) are within the unit circle. The proof is completed.

Remark 3.1: While implementing the consensus protocol (5), we must initialize $\left(\xi_{i}(-k h), \zeta_{i}(-k h)\right), k=1,2, \cdots$, $m+2 ; i \in I$. In the above analysis, it is assumed that $\left(\xi_{i}(-k h), \zeta_{i}(-k h)\right)=\left(\xi_{i}(0), \zeta_{i}(0)\right), k=1,2, \cdots, m+2 ; i \in I$, which does not affect the results obtained in Theorem 3.1.

Remark 3. 2: Although the condition that all the roots of the equation (6) are within the unit circle is necessary and sufficient, for the general communication delay, it is hard to 
derive from this condition the explicit allowable scope of the sampling period that guarantees the multi-agent system (1), applying the consensus protocol (5), to achieve the stationary consensus. Therefore, the results of Theorem 3.1 are mainly used for testing the effectiveness of the sampling period guaranteeing the achievement of stationary consensus.

Remark 3.3: It can be seen from the delay decomposition expression (4) that if $m=0$ and $\varepsilon \neq 0$, then $0<\varepsilon=\tau<h$, i.e., the communication delay is less than the sampling period. In this case, the consensus protocol (5) with the general communication delay degenerates into the consensus protocol with the small communication delay:

$$
u_{i}(t)=\left\{\begin{array}{l}
u_{i}(k h-h)=-\gamma \zeta_{i}(k h-h) \\
+\sum_{j \in N_{i}} a_{i j}\left\{\gamma\left[\xi_{j}(k h-h)-\xi_{i}(k h-h)\right]\right. \\
+(1+\gamma \beta)\left[\zeta_{j}(k h-h)-\zeta_{i}(k h-h)\right] \\
+\beta\left[\left(\zeta_{j}(k h-h)-\zeta_{j}(k h-2 h)\right) / h\right. \\
\left.\left.-\left(\zeta_{i}(k h-h)-\zeta_{i}(k h-2 h)\right) / h\right]\right\}, t \in[k h, k h+\tau) ; \\
u_{i}(k h)=-\gamma \zeta_{i}(k h) \\
+\sum_{j \in N_{i}} a_{i j}\left\{\gamma\left[\xi_{j}(k h)-\xi_{i}(k h)\right]\right. \\
+(1+\gamma \beta)\left[\zeta_{j}(k h)-\zeta_{i}(k h)\right] \\
+\beta\left[\left(\zeta_{j}(k h)-\zeta_{j}(k h-h)\right) / h\right. \\
\left.\left.-\left(\zeta_{i}(k h)-\zeta_{i}(k h-h)\right) / h\right]\right\}, t \in[k h+\tau, k h+h) .
\end{array}\right.
$$

and we have the following corollary:

Corollary 3.1: Consider the second-order multi-agent system (1) with a fixed, undirected and connected network topology. The multi-agent system (1), applying the consensus protocol (24), achieves the stationary consensus if and only if

$$
\begin{gathered}
h^{2} r \lambda_{i}(L)>0, i \in I \backslash\{1\}, \\
h>\tau \beta \lambda_{i}(L), i \in I \backslash\{1\}, \\
{\left[-2 r+2 \tau r \lambda_{i}(L)-2 \lambda_{i}(L)-2 r \beta \lambda_{i}(L)\right] h^{2}} \\
+\left[4+4 \tau r-2 \tau^{2} r \lambda_{i}(L)+4 \tau r \beta \lambda_{i}(L)+4 \tau \lambda_{i}(L)\right. \\
\left.-4 \beta \lambda_{i}(L)\right] h+8 \tau \beta \lambda_{i}(L)>0, i \in I \backslash\{1\}, \\
r \tau \beta \lambda_{i}^{2}(L) h^{2}+\left[-2 \tau^{2} r \beta \lambda_{i}^{2}(L)+2 \tau \beta \lambda_{i}^{2}(L)\right. \\
+2 \tau r \beta^{2} \lambda_{i}^{2}(L)+4 \tau r \beta \lambda_{i}(L)-\tau^{2} r \lambda_{i}(L)+2 \tau \lambda_{i}(L) \\
\left.-2 \beta \lambda_{i}(L)+2 \tau r-2\right] h+\tau \beta \lambda_{i}\left[\tau^{2} r \lambda_{i}(L)-2 \tau \lambda_{i}(L)\right. \\
\left.-2 \tau r \beta \lambda_{i}(L)+2 \beta \lambda_{i}(L)-2 \tau r+2\right]<0, i \in I \backslash\{1\},
\end{gathered}
$$

and

$$
\begin{aligned}
& \left\{-r \tau \beta \lambda_{i}^{2}(L) h^{3}+\left[2+2 r \beta \tau^{2} \lambda_{i}^{2}(L)-2 \tau \beta \lambda_{i}^{2}(L)\right.\right. \\
& -2 r \tau \beta^{2} \lambda_{i}^{2}(L)+r \tau^{2} \lambda_{i}(L)-2 \tau \lambda_{i}(L)-4 r \tau \beta \lambda_{i}(L) \\
& \left.+2 \beta \lambda_{i}(L)-2 \tau r\right] h^{2}+\left[-r \beta \tau^{3} \lambda_{i}^{2}(L)+2 \beta \tau^{2} \lambda_{i}^{2}(L)\right. \\
& \left.\left.+2 r \tau^{2} \beta^{2} \lambda_{i}^{2}(L)-2 \tau \beta^{2} \lambda_{i}^{2}(L)+2 \beta r \tau^{2} \lambda_{i}(L)-2 \tau \beta \lambda_{i}(L)\right] h\right\} \\
& \times\left\{r \tau \beta \lambda_{i}^{2}(L) h^{3}+\left[2-2 r \beta \tau^{2} \lambda_{i}^{2}(L)+2 \tau \beta \lambda_{i}^{2}(L)+2 r \beta^{2} \lambda_{i}^{2}(L)\right.\right. \\
& \left.-r \tau^{2} \lambda_{i}(L)+2 \tau \lambda_{i}(L)+4 r \tau \beta \lambda_{i}(L)-2 \beta \lambda_{i}(L)+2 \tau r\right] h^{2} \\
& +\left[r \beta \tau^{3} \lambda_{i}^{2}(L)-2 \beta \tau^{2} \lambda_{i}^{2}(L)+2 \tau \beta \lambda_{i}(L)-2 r \tau^{2} \beta^{2} \lambda_{i}^{2}(L)\right. \\
& \left.\left.+2 \tau \beta^{2} \lambda_{i}^{2}(L)-2 \beta r \tau^{2} \lambda_{i}(L)\right] h-4 \tau^{2} \beta^{2} \lambda_{i}^{2}(L)\right\}
\end{aligned}
$$

$$
\begin{aligned}
& >2\left[h^{3}-\tau \beta \lambda_{i}(L) h^{2}-\tau^{2} \beta^{2} \lambda_{i}^{2}(L) h+\tau^{3} \beta^{3} \lambda_{i}^{3}(L)\right]\left\{r \lambda_{i}(L) h^{3}\right. \\
& +2\left[r \tau \lambda_{i}(L)-\lambda_{i}(L)-r \beta \lambda_{i}(L)-r\right] h^{2}+2\left[2 r \tau \beta \lambda_{i}(L)+2 \tau \lambda_{i}(L)\right. \\
& \left.\left.-2 \beta \lambda_{i}(L)-\tau^{2} r \lambda_{i}(L)+2 r \tau+1\right] h+6 \tau \beta \lambda_{i}(L)\right\}-\left\{r \tau \beta \lambda_{i}^{2}(L) h^{3}\right. \\
& +\left[-2 \tau^{2} r \beta \lambda_{i}^{2}(L)+2 \tau \beta \lambda_{i}^{2}(L)+2 \tau r \beta^{2} \lambda_{i}^{2}(L)+2 \tau \beta r \lambda_{i}(L)\right. \\
& \left.-\tau^{2} r \lambda_{i}(L)+2 \tau \lambda_{i}(L)+2 \tau r \beta \lambda_{i}(L)-2 \beta \lambda_{i}(L)+2 \tau r\right] h^{2} \\
& +\left[-2 \tau^{2} \beta \lambda_{i}^{2}(L)-2 \tau^{2} r \beta^{2} \lambda_{i}^{2}(L)+2 \tau \beta^{2} \lambda_{i}^{2}(L)-2 \tau^{2} r \beta \lambda_{i}(L)\right. \\
& \left.\left.-4 \tau \beta \lambda_{i}(L)+6 \tau \beta \lambda_{i}(L)+\tau^{3} r \beta \lambda_{i}^{2}(L)\right] h-2 \tau^{2} \beta^{2} \lambda_{i}^{2}(L)\right\} \\
& \times\left\{-r \lambda_{i}(L) h^{4}+\left[2 r \tau \lambda_{i}(L)-2 \lambda_{i}(L)-2 r \beta \lambda_{i}(L)-2 r\right] h^{3}\right. \\
& +\left[-r \tau^{2} \lambda_{i}(L)+2 \tau \lambda_{i}(L)+2 r \tau \beta \lambda_{i}(L)-2 \beta \lambda_{i}(L)+2 \tau r+4\right] h^{2} \\
& +\left[r \beta \tau^{3} \lambda_{i}^{2}(L)-2 \tau^{2} \beta \lambda_{i}^{2}(L)-2 r \tau^{2} \beta^{2} \lambda_{i}^{2}(L)+2 \tau \beta^{2} \lambda_{i}^{2}(L)\right. \\
& \left.\left.-2 r \tau^{2} \beta \lambda_{i}(L)+2 \tau \beta \lambda_{i}(L)\right] h-6 \tau^{2} \beta^{2} \lambda_{i}^{2}(L)\right\}, i \in I \backslash\{1\} .
\end{aligned}
$$

Before proving Corollary 3.1, we need to provide the following lemma.

Lemma 3.1: All the roots of the equation $s^{4}+a_{3} s^{3}+a_{2} s^{2}+a_{1} s+a_{0}=0$, where $a_{0}, a_{1}, a_{2}, a_{3} \in R$, are within the unit circle, if and only if the five inequalities

$$
\begin{aligned}
& 1+a_{3}+a_{2}+a_{1}+a_{0}>0,\left|a_{0}\right|<1, \quad\left|a_{0}^{2}-1\right|>\left|a_{0} a_{3}-a_{1}\right|, \\
& \left|\left(a_{0}{ }^{2}-1\right)^{2}-\left(a_{0} a_{3}-a_{1}\right)^{2}\right|>\mid\left(a_{0}^{2}-1\right)\left(a_{0} a_{2}-a_{2}\right)-\left(a_{0} a_{1}\right. \\
& \left.-a_{3}\right)\left(a_{0} a_{3}-a_{1}\right) \mid \quad \text { and } 1-a_{3}+a_{2}-a_{1}+a_{0}>0 \quad \text { hold }
\end{aligned}
$$
simultaneously( [17]).

The proof of Corollary 3.1 is given below.

Proof: When $0<\varepsilon=\tau<h$, the equation (6) degenerates into

$$
\begin{aligned}
& z^{4}+\left[\left((h-\tau)^{2} r / 2+h+h r \beta-\tau-\tau r \beta+\beta\right.\right. \\
& \left.-\tau \beta / h) \lambda_{i}(L)+(h r-\tau r-2)\right] z^{3}+\left[\left(h^{2} r / 2\right.\right. \\
& -\tau^{2} r+h r \tau-h-h r \beta+2 \tau r \beta+2 \tau-2 \beta+ \\
& \left.3 \tau \beta / h) \lambda_{i}(L)+(1-h r+2 \tau r)\right] z^{2}+\left[\left(\tau^{2} r / 2\right.\right. \\
& \left.-\tau-\tau r \beta-3 \tau \beta / h+\beta) \lambda_{i}(L)-\tau r\right] z+ \\
& (\tau \beta / h) \lambda_{i}(L)=0, i \in I \backslash\{1\} .
\end{aligned}
$$

It follows from Lemma 3.1 that all the roots of the equation (30) are within the unit circle if and only if the inequalities (25), (26), (27), (28) and (29) hold simultaneously. The proof is completed.

\section{Simulations}

In this section we provide the numerical simulations to illustrate the correctness of the above results. Here we consider the second-order multi-agent system (1) with the interconnection topology described in Fig.1. Without loss of generality, assume that the initial states of four agents are chosen as $\xi_{1}(0)=-1, \xi_{2}(0)=-2, \xi_{3}(0)=1, \xi_{4}(0)=2$, $\zeta_{1}(0)=-1, \zeta_{2}(0)=2, \zeta_{3}(0)=1$ and $\zeta_{4}(0)=-2$. Here it is assumed that $\tau=0.15, r=1$ and $\beta=0.1$. 


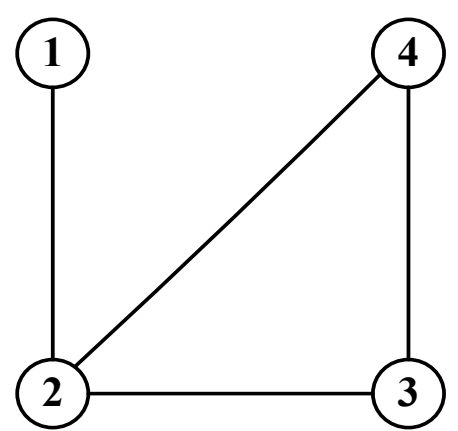

Fig.1. A weighted, undirected and connected graph composed of four agents.

We illustrate the effectiveness of Theorem 3.1 by illustrating the effectiveness of Corollary 3.1. Numerical simulations are shown in Fig. 2 and Fig. 3, respectively.

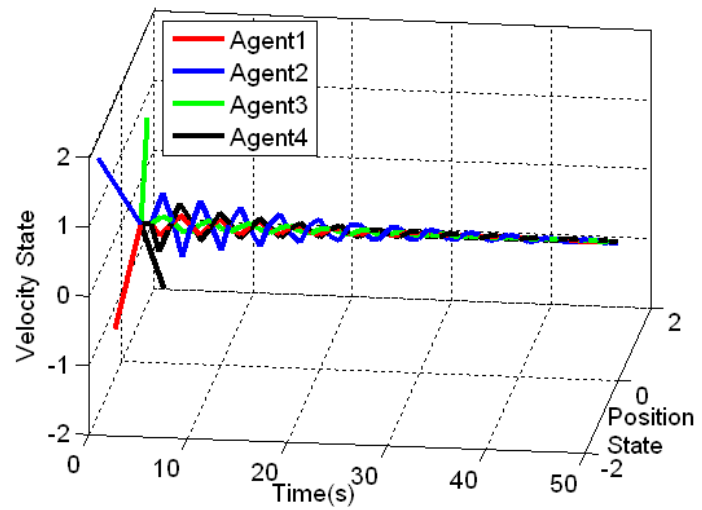

Fig.2. The states of the system (1) applying the protocol (24) with $h=0.3$

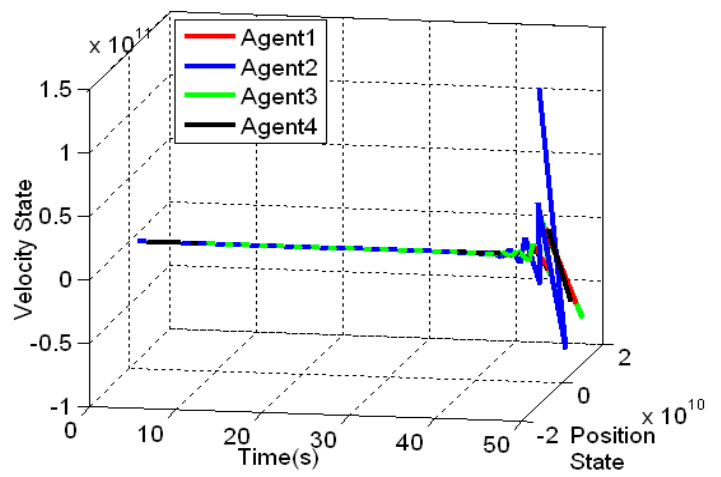

Fig.3. The states of the system (1) applying the protocol (24) with $h=0.7$.

From Fig. 2, we can find that when $h=0.3$, which satisfies the condition given in Corollary 3.1, the given multiagent system (1) applying the consensus protocol (24) achieves the stationary consensus.

From Fig. 3, it can be seen that when $h=0.7$, which does not satisfy the condition given in Corollary 3.1, the given multi-agent system (1) applying the consensus protocol (24) cannot achieve the stationary consensus.

From the above simulations, the effectiveness of Corollary 3.1 is numerically testified, which further illustrates the effectiveness of Theorem 3.1.

\section{Conclusions}

In this paper, we have considered the sampled-data consensus problems of second-order delayed multi-agent systems with delayed-state-derivative feedback. Employing the stability theory of linear systems and algebra graph theory, we have obtained the necessary and sufficient conditions guaranteeing the achievement of stationary consensus. A future work is to extend the results of this paper to the case of second-order multi-agent systems with a directed switching network topology and the time-varying communication delay.

\section{References}

[1] T. Vicsek, A. Czirok, E. B. Jacob, et al., "Novel type of phase transition in a system of self-driven particles," Physical Review Letters, vol. 75, no.6, pp. 1226-1229, 1995.

[2] A. Jadbabaie, A. Lin and A. S. Morse, "Coordination of groups of mobile autonomous agents using nearest neighbor rules," IEEE Transactions on Automatic Control, vol. 48, no. 6, pp. 988-1001, 2003.

[3] W. Ren and R. W. Beard, "Consensus seeking in multi-agent systems under dynamically changing interaction topologies," IEEE Transactions on Automatic Control, vol. 50, no. 5, pp. 655-661, 2005.

[4] R. Olfati-Saber, J. A. Fax and R. M. Murray, "Consensus and cooperation in networked multi-agent systems," Proceedings of the IEEE, vol. 95, no. 1, pp. 215-233, 2007.

[5] W. Ren, R.W. Beard and E.M. Atkins, "Information consensus in multivehicle cooperative control," IEEE Control Systems Magazine, vol. 27, no. 2, pp. 71-82, Apr. 2007.

[6] R. M. Murray, "Recent research in cooperative control of multi-vehicle systems," Journal of Dynamic Systems, Measurement and Control, vol. 129, no. 5, pp. 571-583, 2007.

[7] Y. P. Tian and C. L. Liu, "Robust consensus of multi-agent systems with diverse input delays and asymmetric interconnection perturbations," Automatica, vol. 45, no. 5, pp. 1347-1353, 2009.

[8] Y. C. Cao and W. Ren, "Multi-agent consensus using both current and outdated states with fixed and undirected interaction," Journal of Intelligent \& Robotics Systems, vol. 58, no.1, pp. 95-106, 2010.

[9] Ji. L, S. Xu, Y. Chu and H. Wang, "Distributed average consensus control in networks of agents using outdated states," IET Control Theory and Applications, vol. 4, no. 5, pp. 746-758, 2010.

[10] H. Y. Zhao, S. Y. Xu, and D. M. Yuan, "An LMI approach to consensus in second-order multi-agent systems," International Journal of Control, Automation, and Systems, vol. 9, no. 6, pp. 1111-1115, 2011.

[11] Z. Y. Meng, W. Ren, Y. C. Cao and Z. You, "Leaderless and leaderfollowing consensus with communication and input delays under a directed network topology," IEEE Transactions on Systems, Man, and Cybernetics, Part B: Cybernetics, vol. 41, no. 1, pp. 75-88, 2011.

[12] Z. H. Wu and H. J. Fang, "Improvement for consensus performance of multi-agent systems based on delayed-state-derivative feedback," Journal of Systems Engineering and Electronics, vol. 23, no. 1, pp. 137-144, 2012.

[13] Z. H. Wu and H. J. Fang, "Delayed-state-derivative feedback for improving consensus performance of second-order delayed multi-agent systems," International Journal of Systems Science, vol. 43, no. 1, pp. 140-152, 2012.

[14] H. J. Fang, Z. H. Wu and J. Wei, "Improvement for consensus performance of multi-agent systems based on weighted average prediction," IEEE Transactions on Automatic Control, vol. 57, no.1, pp. 249-254, 2012.

[15] Z. H. Wu, H. J. Fang and Y. Y. She, "Weighted average prediction for improving consensus performance of second-order delayed multi-agent systems," IEEE Transactions on Systems, Man, and Cybernetics-Part B: Cybernetics, vol. 42, no. 5, pp. 1501-1508, 2012.

[16] R. A. Horn and C. R. Johnson, Matrix analysis. Cambridge, U.K.: Cambridge Univ. Press, 1985.

[17] E. I. Jury, "Theory and application of the z-transform method," New York, NY: Wiley Press, 1964. 